\title{
Atomic Structure of Electrochemically Deposited Lithium Metal and Its Solid Electrolyte Interphases Revealed by Cryo-electron Microscopy
}

\author{
Yaobin $\mathrm{Xu}^{1}$, Yang $\mathrm{He}^{1}$, Haiping $\mathrm{Wu}^{2}, \mathrm{Wu} \mathrm{Xu}^{2}$ and Chongmin $\mathrm{Wang}^{1}$ \\ 1. Environmental Molecular Sciences Laboratory, Pacific Northwest National Laboratory, Richland, \\ WA, USA. \\ 2. Energy and Environment Directorate, Pacific Northwest National Laboratory, Richland, WA, USA.
}

Lithium metal is the most attractive anode materials rechargeable batteries due to its low electrode potential and high theoretical specific capacity compared with other present electrode materials. However, the aggressive dendritic growth of $\mathrm{Li}$ metal during cycling makes it too dangerous for practical use. To address this critical issue, we need not only understanding on the macroscopic but also on the microscopic, nanoscopic and atomistic features to probe more fundamental aspects of dendritic growth process. However, current understanding is limited to ensemble studies using low resolution TEM due to both Li metal and the organic liquid electrolyte are unstable under room temperature and electron beam. To overcome these challenges, cryo-transmission electron microscopy (TEM) techniques used in structural biology has been used in battery studies [1-4].

Herein, we applied cryo-transmission electron microscopy (TEM) techniques to characterize the detailed structure of electrochemically deposited Li metal (EDLi) and its solid electrolyte interphase (SEI). Li metal was directly deposited onto the copper TEM grid in a coin cell by applying a current of $2 \mathrm{~mA} \mathrm{~cm}^{-2}$ for 5 mins. 1.2 $\mathrm{M} \mathrm{LiPF}_{6}$ in 3:7 w/w ethylene carbonate/ ethyl methyl carbonate (EC/EMC) with $5 \mathrm{wt} \%$ vinylene carbonate (VC) was added as the electrolyte. After deposition, the TEM grid was taken out from the copper foil and slight rinsed with 1,3-dioxolane (DOL) to remove trace Li salt in the glove box. Then, the TEM grid with deposited Li metal was placed in a sealed bag fulfilled with Ar. The sealed bag was plunged directly into a bath of liquid nitrogen after taken from the Ar-filled glovebox until the Li metal reach to very low temperature. We then quickly took out the copper TEM grid with EDLi from the sealed bag and loaded onto a pre-cooling Gatan cryo-holder using a cryo-transfer station to ensure entire process occurred under cryogenic environment. TEM observations were performed on $300 \mathrm{kV}$ FEI Titan monochromated (scanning) transmission electron microscope equipped with a probe aberration corrector under low dose condition.

Figure 1 (a) is a cryo-TEM image showing the typical morphology of Li metal. It is seen that EDLi exhibit ribbon like configurations with approximately $150 \mathrm{~nm}$ in diameter. Along the edges of the $\mathrm{Li}$ metal a uniform SEI is present with a thickness of $\sim 20 \mathrm{~nm}$. Figure 1 (b) is an atomically resolved TEM images showing the both EDLi, SEI and its interface structures. From the high-resolution image, it is observed that the EDLi contains small crystalline domains dispersed randomly throughout amorphous matrix. Those small crystalline domains were confirmed as $\mathrm{Li}_{2} \mathrm{O}$. It is noted that the interface between EDLi and SEI is not atomically sharp and irregular in shape, there are many small crystalline domains dispersed randomly throughout amorphous SEI matrix as well, based on the area fast Fourier transform (FFT) patterns as shown from the red color-coded regions, we validated the small crystalline domains along the interface are mainly $\mathrm{Li}_{2} \mathrm{CO}_{3}$, while other small crystalline domains are mainly $\mathrm{Li}_{2} \mathrm{O}$. In addition, we also found well crystallinity structures on the surface of the SEI as shown from the purple color-coded region, which was determined to be large grains of $\mathrm{Li}_{2} \mathrm{O}$ with clear lattice fringes, and those large $\mathrm{Li}_{2} \mathrm{O}$ grains has same zone axis of [110]. Figure 1 (c) is the schematic of the observed SEI 
structure formed on EDLi in VC electrolyte.

The presentation will also cover ongoing investigations and data analysis; all of which may provide further insights into the understanding of the correlation between electrolyte chemistry and Li metal dendrite growth kinetics and morphology. We believe these will be helpful in designing next generation advanced rechargeable batteries [5].

\section{References:}

[1] YZ Li et al, Science. 358 (2017), p. 506.

[2] XF Wang et al, Nano Lett. 17 (2017), p. 7606.

[3] MJ Zachman et al, Nature. 560 (2018), p. 345

[4] XF Wang, YJ Li and YS Meng, Joule. 2 (2018), p. 1.

[5] The work was conducted in the William R. Wiley Environmental Molecular Sciences Laboratory (EMSL), a national scientific user facility sponsored by DOE's Office of Biological and Environmental Research and located at PNNL.
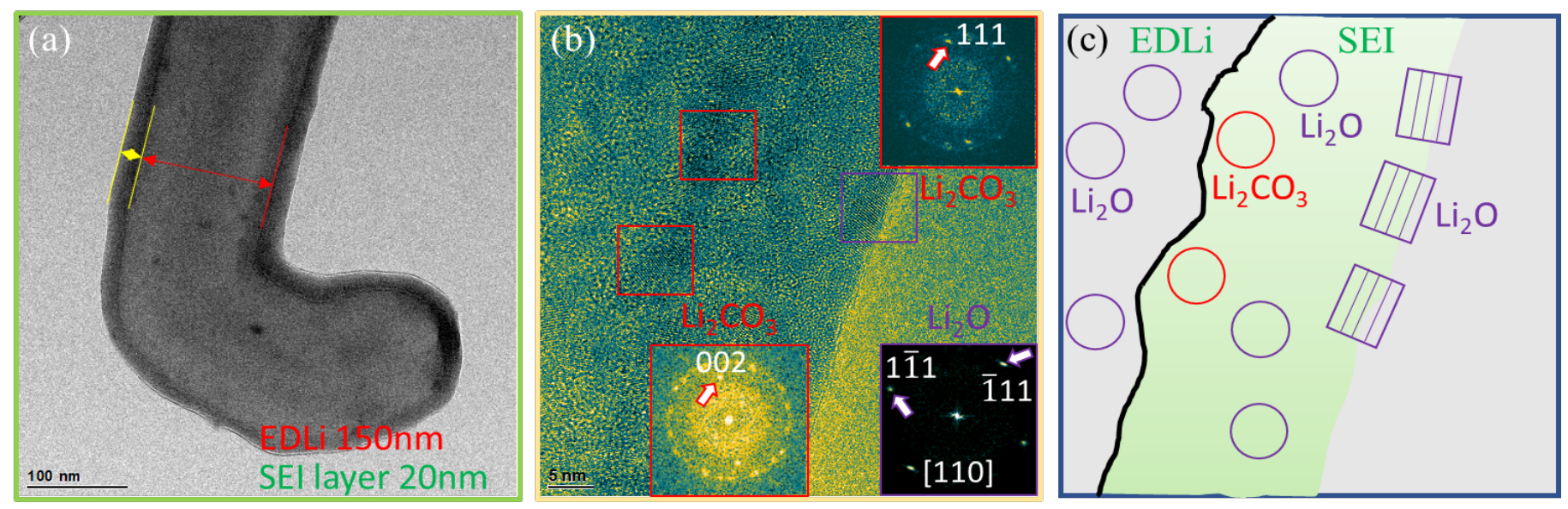

Figure 1. Atomic-resolution TEM of electrochemically deposited Li metal (EDLi) and SEI interface. (a) Bright-field TEM image of the EDLi at a low magnification. (b) Atomic-resolution image of the EDLi and SEI interface. (c) Schematic of the observed SEI formed on EDLi. 